\title{
Subcutaneous abscess due to Pyrenochaeta romeroi in a renal transplant recipient
}

\author{
Yuen Yue Candice Chan ${ }^{1,2}$ MBChB, BSc, Ai Ling $\operatorname{Tan}^{1,2}$, MBBS, FRCPA, Ban Hock $\underline{\operatorname{Tan}}^{1,2}$, MBBS, FRCP
}

\begin{abstract}
An infective aetiology, including fungal infection, should be considered in the differential diagnosis of immunocompromised patients presenting with skin lesions. Dematiaceous fungi are recognised as pathogens in organ transplant recipients. Herein, we describe a rare case of a chronic necrotising granulomatous skin lesion caused by Pyrenochaeta romeroi in a renal transplant recipient, and review the existing literature on the topic. To the best of our knowledge, this is the first report of such a case in Singapore. Recognition of infections caused by dematiaceous fungi is important because some strains are difficult to identify and require special molecular diagnostic techniques. Treatment involves surgical excision and long-term antifungal therapy. Data on the optimal antifungal regimen in such a diagnosis is limited.
\end{abstract}

Keywords: eumycetoma, fungi, itraconazole, Pyrenochaeta romeroi, renal transplant

\section{INTRODUCTION}

Pyrenochaeta romeroi ( $P$. romeroi) is a saprophytic fungus widely distributed in the environment; it can be found on wood and in plants. ${ }^{(1)}$ The fungal spores of $P$. romeroi can be inoculated into deeper tissues by trauma, ${ }^{(2,3)}$ and are a rare cause of skin and subcutaneous tissue infections. Herein, we report Singapore's first case of $P$. romeroi infection involving a 55-year-old renal transplant recipient who developed a chronic necrotising granulomatous skin lesion. The challenges involved in the diagnosis and treatment of this uncommon disease is discussed in this report.

\section{CASE REPORT}

A 55-year-old Chinese man presented to our institution with a slow growing, painless nodular lesion on his left posterior thigh. One year before the current presentation, the patient had undergone a living related donor renal transplantation. For the transplantation, the patient received cyclosporine and thymoglobulin at induction, followed by maintenance immunosuppressive therapy with prednisolone $10 \mathrm{mg}$ once daily, mycophenolate mofetil $250 \mathrm{mg}$ twice daily, and cyclosporin $45 \mathrm{mg}$ once daily. He had an uneventful postoperative recovery, and his transplanted kidney was found to be functioning well with no signs of rejection during follow-up. He did not have diabetes mellitus or a medical history of note, and he denied any history of trauma, soil exposure or participation in gardening activities. Throughout that one year (from renal transplantation to the current presentation), the patient did not show any systemic symptoms and his inflammatory markers (white cell count and C-reactive protein) were unremarkable. For his current presentation, the patient underwent ultrasonography of his left posterior thigh lesion, which revealed a thick-walled cystic mass.

Histologic examination of the biopsied specimen showed necrotising granulomatous inflammation with fungal hyphae. The thigh lesion recurred and progressed despite repeated attempts at complete surgical excision over a one-year period. Diagnostic specimens for fungal cultures were obtained by means of surgical excision or needle biopsy. The specimens were inoculated onto Sabouraud dextrose agar and Sabouraud dextrose agar with chloramphenicol, and incubated at $30^{\circ} \mathrm{C}$. Five specimens grew mould that were non-sporulating after approximately five days of incubation. In view of the repeated isolation of non-sporulating mould, polymerase chain reaction (PCR) sequencing of the internal transcribed spacer region of ribosomal RNA gene was performed. PCR was consistently positive for $P$. romeroi (GenBank Accession no. EF488398.1) in three of the five clinical samples, with a $100 \%$ identity on NCBI BLAST analysis. Repeat bacterial and mycobacterial testings failed to yield organisms.

In vitro antifungal susceptibility test was performed, but the results were inconclusive because the fungus failed to grow well in the susceptibility testing medium. Nevertheless, the interpretation of the susceptibility data of this unusual mould ( $P$. romeroi) would be difficult because there was no standardised susceptibility data for moulds other than those of the Aspergillus genus. The patient was started empirically on itraconazole syrup $200 \mathrm{mg}$ twice daily for a period of two years. There was gradual reduction of the lesion size and the lesion remained quiescent in those two years. Throughout the course of treatment, itraconazole serum level was not monitored due to the unavailability of the test at our centre.

${ }^{1}$ Department of Infectious Diseases, ${ }^{2}$ Department of Pathology, Singapore General Hospital, Singapore

Correspondence: Dr Chan Yuen Yue Candice, Registrar, Department of Infectious Diseases, Singapore General Hospital, Outram Road, Singapore 169608, Candice.chan.y.y@singhealth.com.sg 
In view of the patient's worsened renal function and increased serum cyclosporine level due to drug-drug interactions, his renal physician changed his treatment with itraconazole syrup to itraconazole capsules and reduced his dose to $200 \mathrm{mg}$ once daily. However, the lesion's size increased with the dose reduction; this was rectified with the resumption of itraconazole syrup $200 \mathrm{mg}$ twice daily. The patient passed away three years later in 2010 from an acute myocardial infarction.

\section{DISCUSSION}

Eumycetoma, a chronic subcutaneous infection caused by fungi, results in a granulomatous inflammatory response in the deep dermis and subcutaneous tissue, which may extend to the underlying bone. ${ }^{(3)}$ Members of the anamorphic genus Pyrenochaeta are dematiaceous (i.e. dark-coloured), filamentous fungi that inhabit soil and plant debris, particularly in tropical climates..$^{(2,4-7)}$ They are pathogens of various plant species and rarely cause human infections; when human infection occurs, it is usually due to the introduction of fungal spores into deeper tissues by trauma, typically in exposed areas of the body. ${ }^{(3,6,7)} P$. romeroi has been isolated from cases of mycetoma, in which the fungi is characterised by the production of soft, irregular, black grains with a subhyaline center. ${ }^{(2,4,5)}$ In those cases, the lesions were found to develop slowly after trauma and were typically localised to cutaneous and subcutaneous tissues.

In cases of Pyrenochaeta infection, inflammatory markers including leucocyte count and C-reactive protein are typically normal, ${ }^{(1,7)}$ as was seen in the present case. Most of the reported cases of $P$. romeroi infection involved mycetoma patients with a history of residence in India, Senegal or Pakistan. ${ }^{(7-9)}$ Patients included healthy individuals, renal transplant recipients on immunosuppressive therapy, and acute lymphoblastic leukaemia patients on chemotherapy. ${ }^{(1,6-9)}$ The identification of Pyrenochaeta species is difficult because some strains do not produce characteristic diagnostic structures in culture and because there is limited expertise available in most diagnostic microbiology laboratories. Since there is no standardised therapy for eumycetoma, the main treatment is surgical excision.

Data on the antifungal susceptibility of the Pyrenochaeta species remains scarce due to the low availability and number of clinical isolates, difficulty in growing the fungus in culture, and little knowledge about the relation between the minimal inhibitory concentration (MIC) and clinical outcome of Pyrenochaeta infection. ${ }^{(7,8)}$ Thiyagarajan et al and Badali et al reported that isavuconazole, posaconazole, terbinafin and ketoconazole have potent in vitro activity against $P$. romeroi..$^{(1,7)}$ $P$. romeroi has been reported to be resistant, with high MICs, to amphotericin $\mathrm{B}$, fluconazole and caspofungin, ${ }^{(7,8)}$ and the effect of voriconazole was reported to be variable. ${ }^{(1,6-8)}$ Itraconazole was shown to be potent by Badali et al, but resistant by Cerar et al. ${ }^{(7,8)}$ Since the susceptibility test was unsuccessful in the present case, the choice of itraconazole was empirical.
Our patient responded to the treatment with itraconazole after prolonged therapy, but relapsed during dose reduction.

Khan et al and Badali et al reported two cases of subcutaneous infections that were successfully treated with surgical excision alone (i.e. without the use of antifungals). ${ }^{(6,7)}$ Other reports used surgical debridement followed by prolonged use of triazole. ${ }^{(1,8)}$ In a case series of patients with phaeohyphomycosis, itraconazole was demonstrated to be effective in some patients. ${ }^{(10)}$ Cerar et al reported the case of an immunocompetent patient with $P$. romeroi osteomyelitis who failed to respond to the combination of itraconzole (200 mg twice daily) and flucytosine ( $1 \mathrm{~g}$ three times daily), but responded to 22 months of posaconazole therapy. ${ }^{(8)}$ Thiyagarajan et al reported the case of a renal transplant recipient with subcutaneous $P$. romeroi infection who responded to a twomonth treatment with voriconazole after surgical excision, with no recurrence. ${ }^{(1)}$ The present case adds to the evidence provided by the aforementioned case series, ${ }^{(10)}$ which found that prolonged itraconazole therapy leads to improvement in those who fail to respond to surgical treatment alone. In the present case, the failure to respond surgical treatment alone may be the result of our patient's immunocompromised state.

There are several reasons as to why the isolation of $P$. romeroi in our patient is likely to be clinically significant and unlikely due to colonisation or contamination. Firstly, the fungus was grown from the lesion from the left thigh on five occasions, and all specimens were obtained by surgical biopsy or needle aspiration of the lesion. Although the fungus was non-sporulating and a diagnosis could not be made based on the fungal cultures, subsequent molecular diagnostic sequencing of three of the five isolates, performed on separate occasions, all yielded P. romeroi. Finally, the lesion decreased in size only after the patient was treated with itraconazole, and this reduction in size was reversed when the dose of itraconazole was reduced.

In post-transplant or immunocompromised patients with skin and soft tissue infections, it is imperative to consider a fungal aetiology. An early diagnosis may be possible via a biopsy of the lesion. P. romeroi infection is rare, difficult to identify, and knowledge about its antifungal susceptibility is limited. Although treatment of eumycetoma is mainly surgical, we recommend that additional antifungal therapy and close follow-up be practised when immunosuppressed patients are involved. This is so that further progression of the infection and recurrence of this invasive disease are prevented. In the present case, itraconazole appears to be effective against eumycetoma that is caused by $P$. romeroi infection.

\section{REFERENCES}

1. Thiyagarajan UM, Bagul A, Nicholson M. A nodulo-cystic eumycetoma caused by Pyrenochaeta romeoi in a renal transplant recipient: a case report. J Med Case Reports 2011; 5:460.

2. André $M$, Brumpt V, Destombes $P$, Segretain $G$. [Fungal mycetoma with black grains due to Pyrenochaeta romeroi in Cambodia]. Bull Soc Pathol Exot Filiales 1968; 61:108-12. French. 
3. Maiti PK, Haldar PK. Mycetomas in two different trauma-prone parts of body: a study of 212 cases. Indian J Med Microbiol 1998; 16:19-22.

4. Serrano JA, Pisano ID, Lopez FA. Black brain minimycetoma by Parenochaeta mackinnonii. The first clinical case of eumycetoma reported in Barinas state, Venezuela. Clinical-histological features and case treatment. J Mycol Med 1998; 8:34-9.

5. Borelli D. [Opportunistic fungi as producers of gray colonies and mycetomata]. Dermatologica 1979; 159 (Suppl 1):168-74. French.

6. Khan Z, Ahmad S, Kapila K, et al. Pyrenochaeta romeroi: a causative agent of phaeohyphomycotic cyst. J Med Microbiol 2011; 60:842-6.
7. Badali H, Chander J, Gulati N, et al. Subcutaneous phaeohyphomycotic cyst caused by Pyrenochaeta romeroi. Med Mycol 2010; 48:763-8.

8. Cerar D, Malallah YM, Howard SJ, Bowyer P, Denning DW. Isolation, identification and susceptibility of Pyrenochaeta romeroi in a case of eumycetoma of the foot in the UK. Int J Antimicrob Agents 2009; 34: 617-8.

9. Girard C, Dereure O, Rispail P, Durand L, Guilhou JJ. Subcutaneous phaeohyphomycosis due to Pyrenochaeta romeroi in a patient with leprosy. Acta Derm Venereol 2004; 84:154-5.

10. Sharkey PK, Graybill JR, Rinaldi MG, et al. Itraconazole treatment of phaeohyphomycosis. J Am Acad Dermatol 1990; 23:566-86. 